\title{
Resolving intraluminal drug and formulation behavior: Gastrointestinal concentration profiling in humans
}

\author{
Joachim Brouwers*, Patrick Augustijns \\ Drug Delivery and Disposition, KU Leuven, Leuven, Belgium
}

\section{A R T I C L E I N F O}

Article history:

Received 5 December 2013

Received in revised form 21 January 2014

Accepted 23 January 2014

Available online $\mathrm{xxxx}$

\section{Keywords:}

Intestinal absorption

Intraluminal drug and formulation behavior

Gastrointestinal drug concentrations

\begin{abstract}
A B S T R A C T
Despite the wide use of the oral route to deliver systemic drugs to humans, the intestinal absorption process is still not fully understood. Especially for complex absorption-enabling strategies (e.g. solubilization, supersaturation, etc.), the in vivo performance is difficult to predict. Considering the current share of drug candidates that suffer from a non-favorable absorption potential and therefore rely on these strategies, there is a growing interest in approaches that aim to resolve the multitude of interactions between drugs, formulation factors and the gastrointestinal environment. In this respect, gastrointestinal concentration profiling following drug administration to humans is a recent but promising strategy that complements more established techniques including gastrointestinal imaging. In the present review, a number of case studies will be discussed to demonstrate the added value of gastrointestinal concentration profiling to gain in-depth knowledge of intraluminal drug and formulation behavior and to identify those processes key for drug absorption. Examples include a better understanding of intestinal precipitation of weakly basic drugs, clarifying inter-individual or food-induced variability in absorption, and an improved insight into the solubility-permeability interplay. As manifested in a recently initiated European project on oral biopharmaceutics tools (OrBiTo), intraluminal concentration profiling will contribute to the development of relevant simulation models that are built upon a solid understanding of human drug and formulation behavior, and allow for a more predictive in vitro and in silico evaluation of absorption.
\end{abstract}

(c) 2014 Elsevier B.V. All rights reserved.

\section{Introduction}

Impaired intestinal absorption, causing insufficient or variable bioavailability, is one of the key reasons for suboptimal clinical performance of orally administered drugs with systemic action. Reliable preclinical prediction of absorption during drug candidate selection and formulation development is of paramount importance to tackle absorption issues and reduce drug attrition during clinical trials. Classically, in vitro evaluation of absorption involves assessment of the drug's absorption potential (i.e. solubility and permeability) and, in the course of formulation development, dissolution in static, one compartment setups (USP apparatus I and II). While these approaches may suffice to forecast uptake of easily absorbed molecules, they are less accurate as in vivo predictive tools for molecules with less favorable absorption potential (i.e. limited solubility and/or permeability). Enabling absorption of

\footnotetext{
* Corresponding author. Address: Drug Delivery and Disposition, KU Leuven, Gasthuisberg ON 2, Herestraat 49, Box 921, KU Leuven, Belgium. Tel.: +32 16330 w310.

E-mail address: joachim.brouwers@pharm.kuleuven.be (J. Brouwers).
}

these compounds requires sometimes challenging formulation strategies of which the performance greatly depends on the gastrointestinal physiology and the interplay between multiple processes, as schematically represented in Fig. 1. Attempts to capture this interplay in biorelevant in vitro evaluation tools have resulted in the development of dynamic, multicompartmental systems to account for time-dependent fluid composition, gastrointestinal transit and/or permeation (e.g. USP apparatus IV, dissolution-permeation system, TNO Intestinal Model) (McAllister, 2010). In addition, computational physiologically based models have been anticipated as powerful tools to integrate in vitro data and simulate the human absorption process (physiology-based (PBPK) pharmacokinetic modeling) (Kostewicz et al., in press). For many drug-formulation combinations, however, the predictive power of these models still appears either insufficient or uncertain (Kostewicz et al., in press).

Further model optimization is often impeded by gaps in the knowledge on the in vivo behavior of complex formulations in the gastrointestinal tract. An example that currently receives much attention is the precipitation of drugs in the stomach or intestine. Since the induction of intraluminal supersaturation has recently 


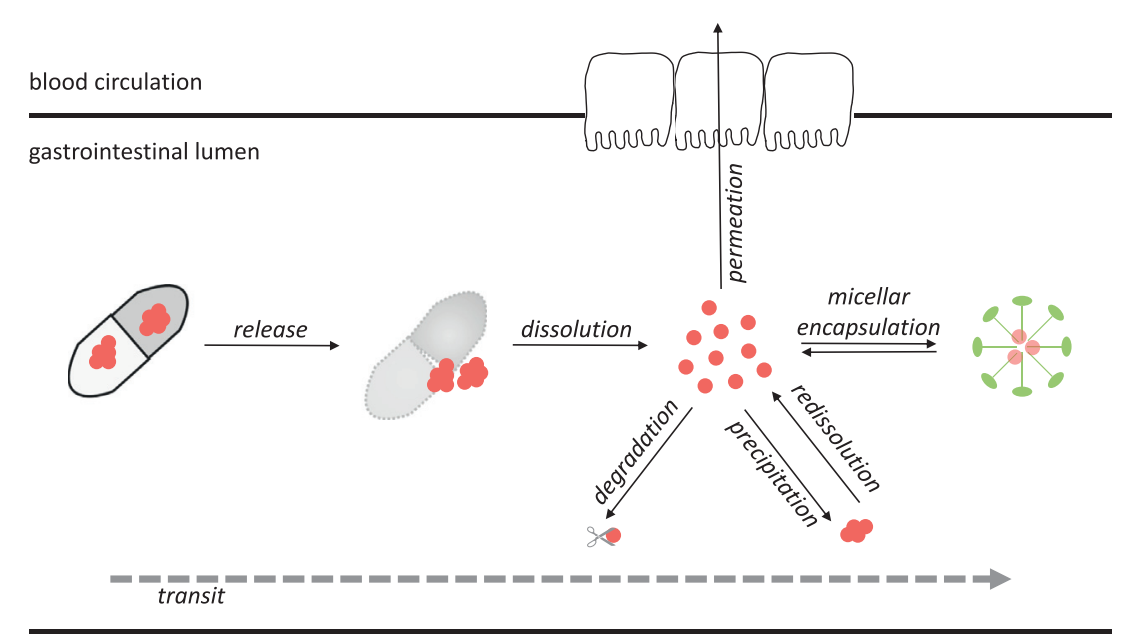

Fig. 1. Schematic and simplified representation of intraluminal processes determining intestinal absorption.

become a promising and widely investigated formulation strategy to enable absorption of poorly soluble drugs, assessment of possible precipitation prior to drug uptake is crucial to evaluate the potential of supersaturating drug delivery systems. However, the knowledge on in vivo precipitation in the gastrointestinal tract is nearly non-existing and recent studies suggest that most in vitro simulations of precipitation are inaccurate (Bevernage et al., 2013). To overcome these types of obstacles, reference studies that assess the intraluminal fate of drugs and dosage forms in humans are receiving intensified attention. This has recently been manifested within the Oral Biopharmaceutics Tools (OrBiTo) project. OrBiTo, a new project within the Innovative Medicines Initiative (IMI) between the European Union and the European Pharmaceutical Industry Association (EFPIA), pursues the development of novel, in vivo predictive experimental and theoretical models for oral absorption (Lennernäs et al., in press). One of the main pillars of OrBiTo is a thorough understanding of the absorption process that should support these novel tools.

In this framework, the present review aims to demonstrate the assessment of gastrointestinal concentrations upon oral drug administration as a recent tool to evaluate intraluminal drug and formulation behavior in humans and to guide the optimization of simulation models.

\section{Evaluating gastrointestinal drug and formulation behavior in humans}

Traditionally, the development of in vitro models that aim to simulate intestinal drug absorption is driven by reference data from classic pharmacokinetic studies. This implies, at best, indirect assessment of the underlying intraluminal processes through modeling of systemic concentration-time profiles using, for instance, deconvolution or PBPK modeling. It has been recognized that only direct evaluation of the intraluminal behavior allows for an indepth understanding of the complex interplay between gastrointestinal physiology and drug formulations. For this purpose, both imaging and intubation techniques can be used.

\subsection{Imaging techniques}

Imaging of the gastrointestinal tract allows for a non-invasive way to follow the fate of orally administered dosage forms without disturbing physiological processes. The historical gold standard is gamma scintigraphy, but more recently, alternative approaches have been introduced based on magnetic tracking principles. In this paper, the different techniques, including their strengths and weaknesses, are only shortly raised; for a detailed discussion, we refer to recent reviews on this topic (Corá et al., 2011; Weitschies and Wilson, 2011; Weitschies et al., 2010).

Gamma scintigraphy has been applied to investigate the gastrointestinal behavior of pharmaceutical dosage forms since 1976 (Casey et al., 1976; Wilding et al., 2001). In this method, a dosage form is labeled with a gamma-emitting radioisotope, either by direct incorporation during formulation $\left({ }^{99 \mathrm{~m}} \mathrm{Tc}\right.$ or $\left.{ }^{111} \mathrm{In}\right)$ or by neutron activation of a stable isotope (e.g. ${ }^{152} \mathrm{Sm}$, activated to ${ }^{153} \mathrm{Sm}$ ). Subsequently, the distribution of these isotopes in the gastrointestinal tract can be assessed by means of a scintillation camera, which generates a two-dimensional projected view. This allows monitoring the intraluminal location of the labeled dosage form (transit) and its integrity (release) over the entire length of the gastrointestinal tract. The approach has been used to evaluate the residence time in the stomach, small intestine and colon in different conditions (e.g. fasted versus fed state). Furthermore, the combination of scintigraphy with pharmacokinetic studies (pharmacoscintigraphy) has become an important means of increasing insight into the impact of transit and release of oral dosage forms on drug absorption.

The main disadvantage of scintigraphy is the necessity to apply ionizing radiation, requiring essential precautions and regulatory issues. Alternative imaging techniques that avoid this type of strain to volunteers and investigators are based on the detection of magnetically labeled dosage forms (Corá et al., 2011), including the method of magnetic marker monitoring (MMM) (Weitschies et al., 2010). For MMM, the dosage form is marked as a permanent magnetic dipole by the incorporation of ferromagnetic material and subsequent magnetization in a strong magnetic field. After oral administration, the three dimensional localization of the dosage form (i.e. the magnetic source) in the gastrointestinal tract can be monitored as a function of time. Compared to scintigraphy, the high temporal (real-time) and spatial $(1 \mathrm{~mm})$ resolution of MMM allows for a more detailed insight into gastrointestinal transit. In addition, disintegration of the dosage form can be quantitatively monitored, as it is related to a decrease in magnetic strength. Once released, however, particles cannot be visualized anymore.

\subsection{Intubation techniques}

Catheters introduced through the nose, mouth or rectum allow for direct access to the contents of the gastrointestinal tract of human volunteers. Apart from aspirating gastric and intestinal fluids 
that can be used as reference media for optimization of simulating fluids, intubation enables monitoring drug concentrations in gastrointestinal fluids to study absorption processes. Initial studies focused on distinct processes (e.g. absorption, dissolution, metabolism) by perfusing an intestinal segment with a drug solution or suspension. This approach was illustrated for the first time by Jobin et al. (1985). Using multi-lumen tubes, a drug solution was perfused in different regions of the intestinal tract. Drug absorption was monitored based on the disappearance of the drug from aspirated intestinal fluid and the appearance in blood. A similar but refined approach is the Loc-I-Gut ${ }^{\circledR}$ perfusion method, originally proposed to investigate the permeation of drugs across the intestinal epithelium in vivo (Knutson et al., 1989, 2009). In this technique, one multi-lumen tube is used; two latex balloons are inflated in order to isolate an intestinal segment that is subsequently perfused with a drug solution. Permeability is calculated from the disappearance of drug during perfusion. This approach has become the standard method to determine human intestinal permeability (Lennernäs, 1998). The Loc-I-Gut ${ }^{\circledR}$ method also allows assessing intestinal drug metabolism and luminal excretion (Petri et al., 2003; Tannergren et al., 2003), and performing in vivo dissolution tests when the segment is perfused with a drug suspension (Bønløkke et al., 1997). Observed differences between in vitro and in vivo dissolution of drugs indicate the impact of the complex and dynamic gastrointestinal environment (Bønløkke et al., 1999, 2001).

In 2005, Brouwers et al. investigated the intraluminal behavior of two theophylline formulations in healthy volunteers by determining drug concentrations in simultaneously aspirated duodenal and jejunal fluids (Brouwers et al., 2005). For the first time, intraluminal drug concentrations were monitored as a function of time upon oral drug intake in humans. Since then, additional studies with more challenging drugs and formulations have been performed, illustrating that this approach offers a unique way to gain insight into intraluminal dosage form performance and subsequent drug absorption. In the following sections of this review, we will discuss the methodology and the added value of monitoring intraluminal drug concentrations in humans to understand gastrointestinal formulation behavior and to guide optimization of simulation models.

\section{Gastrointestinal concentration profiling upon drug administration in humans: methodology}

The assessment of gastrointestinal drug concentrations in humans is schematized in Fig. 2. This section comprises a brief description of different methodological aspects involved in this type of clinical studies.

\subsection{Intubation of healthy volunteers}

In all studies reported so far, fluids were aspirated from the upper gastrointestinal tract of healthy volunteers. In one study, the previously mentioned Loc-I-Gut ${ }^{\circledR}$ catheter was employed to aspirate fluids from a semi-open intestinal segment (created by inflating the distal balloon of the catheter) (Bergman et al., 2007). Other studies sampled from open segments, making use of standard double-lumen catheters designed to aspirate gastric and intestinal fluids in clinical practice. The catheters are introduced via the mouth or nose and positioned into the stomach (usually antrum), duodenum or proximal jejunum. Positioning is guided by a wire and checked by means of fluoroscopy (X-rays). This implies that this type of study cannot be performed in pregnant women or children. In a number of studies, two double-lumen catheters have been used to simultaneously assess drug concentrations at two intraluminal positions: duodenum and jejunum (Brouwers et al., 2005, 2006), or stomach and duodenum (Brouwers et al., 2007a; Walravens et al., 2011). The latter positioning is shown in the X-ray picture in Fig. 3.

In contrast to imaging techniques, intubation studies are invasive and may, as such, disturb the gastrointestinal physiology. A number of reports, however, indicated only minor or non-significant effects of transpyloric tubes on gastric emptying, gastric secretions and duodenogastric reflux (Go et al., 1970; Longstreth et al., 1975; Näslund et al., 2000; Read et al., 1983; Rees et al., 1979). Read et al. (1983) did observe a significant reduction in the small intestinal transit time but this may be related to the use of a long tube, reaching the terminal ileum.

\subsection{Dosage form administration}

Drugs are administered either orally (dosage form with water) (Brouwers et al., 2005, 2006, 2007a; Walravens et al., 2011) or directly into the stomach by means of a catheter (solution or suspension) (Bergman et al., 2007; Psachoulias et al., 2011; Vertzoni et al., 2012). Obviously, 'real-life' administration is most relevant, but gastric dosing may be more appropriate for mechanistic purposes. For instance, to investigate intestinal supersaturation and precipitation of weakly basic drugs upon gastric emptying, it might be advantageous to neglect gastric dissolution by administering drug solutions directly into the stomach (Psachoulias et al., 2011). Fed state conditions can be simulated by either a nutritional drink (Brouwers et al., 2007a) or a homogenized liquid meal (Vertzoni et al., 2012). It should be recognized that solid meals will have a different effect on gastric motility, but they are incompatible with aspiration studies (clogging of catheter).

\subsection{Sampling and characterizing gastrointestinal fluids}

Once the drug has been administered, gastrointestinal fluids are manually aspirated as a function of time, typically in intervals of 530 min during a period of 2-4 h. Immediately upon sampling, precautions should be taken to avoid any post-sampling alterations in the aspirated fluids. Enzymatic digestion should be inhibited by adding lipase and protease inhibitors, especially when investigating fed state conditions. In case of poorly soluble drugs, it is essential to separate non-dissolved and/or precipitated drug particles (centrifugation or filtration) and to avoid possible further precipitation by diluting the particle-free sample. Also for degradationsensitive drugs (enzymatic and/or chemical), the required precautions should be taken. During handling, care should also be taken to limit exposure to atmospheric $\mathrm{CO}_{2}$, since this may affect the $\mathrm{pH}$ of the poorly buffered aspirates (Kalantzi et al., 2006; Perez de la Cruz Moreno et al., 2006). Once the samples have been stabilized, they can be stored prior to further analysis.

Quantification of drug concentrations in the aspirated fluids requires validated bioanalytical assays employing common techniques for sample preparation (typically protein precipitation or extraction), compound separation (HPLC) and compound detection (UV, fluorescence or MS/MS). Considering the high variability of the composition of gastrointestinal fluids, the bioanalytical method has to be robust against matrix effects. It should further be noted that drugs dissolved in intestinal fluids can be present in multiple colloidal phases, including a lipid phase and a water phase containing micelles and vesicles. Especially in fed state conditions, it may be highly informative to utilize ultracentrifugation to separate at least the lipid phase from the micelle-containing water phase, as illustrated by Vertzoni et al. (2012).

Apart from drug concentrations, additional characteristics of the aspirated fluids may be determined to further improve our understanding of intraluminal formulation behavior. In case of 


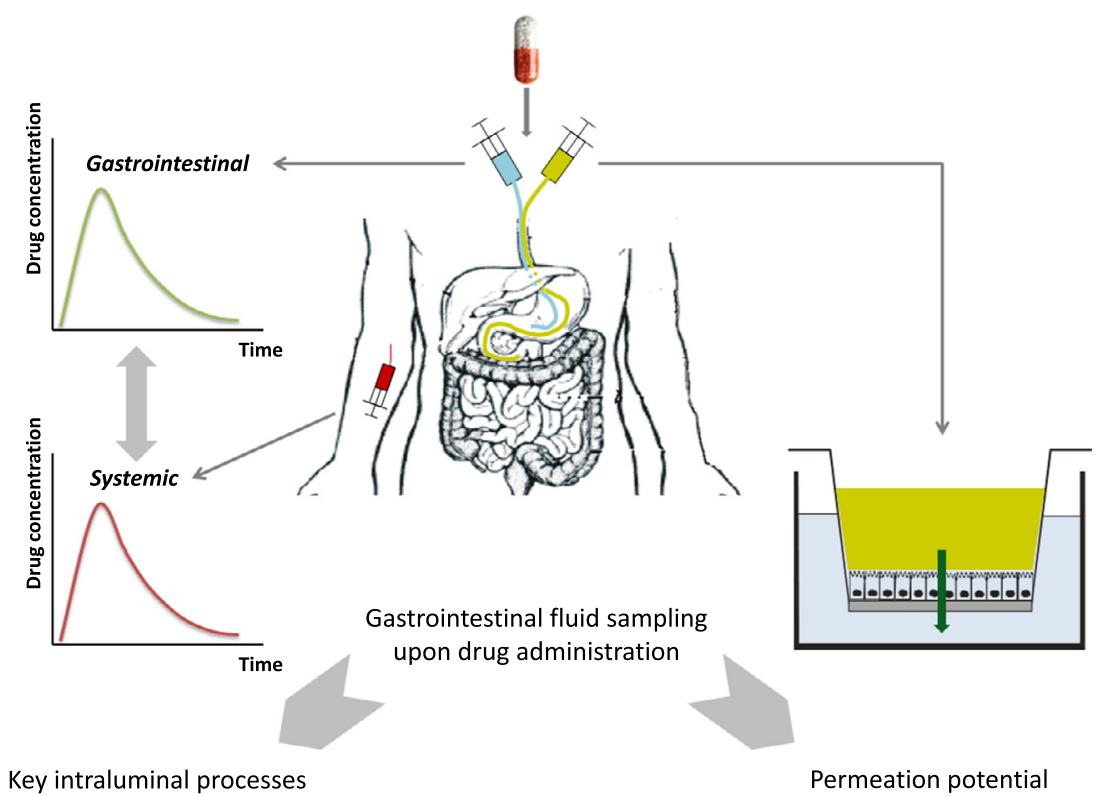

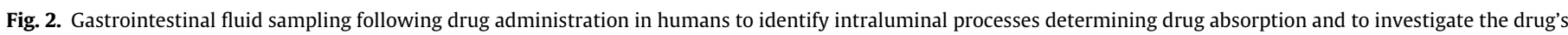
permeation potential.

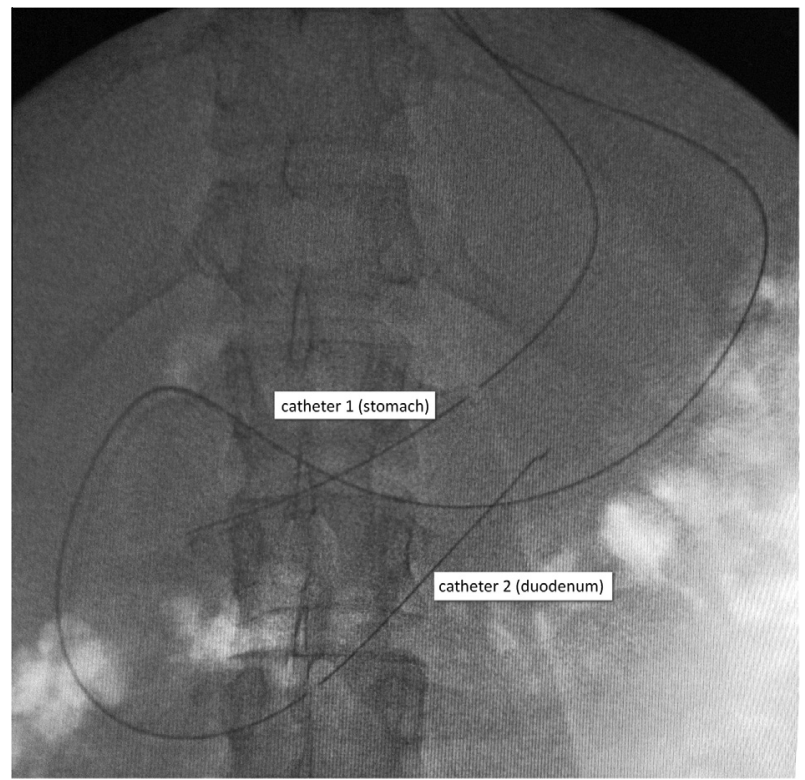

Fig. 3. Fluoroscopic image of the position of two double-lumen catheters in the gastrointestinal tract of a healthy volunteer.

intestinal drug precipitation, for instance, the solid-state characteristics of the precipitate have been assessed by means of X-ray powder diffraction (XRPD) (Psachoulias et al., 2011) or Raman microscopy (Bergman et al., 2007) since they may determine the rate of redissolution. In addition, the aspirated fluids may be used as donor media in in vitro permeation models (Brouwers et al., 2006; Vertzoni et al., 2012). This gains highly relevant insight into the complex process of drug permeation from intraluminal fluids. Finally, fluid properties including $\mathrm{pH}$, osmolality, viscosity, surface tension, buffer capacity and concentration of bile salts, phospholipids and dietary (digestion) products, may be determined for correlation purposes.

With respect to the sampling of gastrointestinal fluids, it is important to recognize that both the stomach and the upper small intestine are far from homogeneous systems (Koziolek et al., 2013; Schiller et al., 2005). Although this may cause artefactual variability in the concentration-time profiles, it did not compromise the studies performed so far (see Section 4).

\subsection{Relating intraluminal behavior to drug absorption}

Gastrointestinal concentration-time profiles of orally administered drugs reflect the interplay between multiple gastrointestinal processes (transit, release, dissolution, precipitation, degradation and mucosal permeation) and provide as such insight into the intraluminal behavior of dosage forms. From a pragmatic point of view, it is essential to identify those intraluminal processes and variables that are key for drug absorption (and thus formulation performance). This can be achieved by the simultaneous sampling of gastrointestinal fluid and blood to correlate intraluminal and systemic concentrations, as will be illustrated in the examples below. Obviously, determining systemic drug concentrations only makes sense if simultaneously aspirated volumes of gastric and intestinal fluids are kept to a minimum, in order to minimize the effect of drug sampling on the absorption process.

\section{Applications of gastrointestinal concentration profiling in humans}

Case studies presented in the following sections will demonstrate how gastrointestinal drug concentration profiling has improved our understanding of selected aspects of intraluminal drug and formulation behavior in humans. Also the optimization of in vitro simulation models based on this novel type of knowledge will be illustrated.

\subsection{Intraluminal solubilization and the solubility-permeability interplay}

The HIV protease inhibitor amprenavir is a lipophilic drug $(\log P \approx 2)$ with limited aqueous solubility $(\approx 70 \mu \mathrm{g} / \mathrm{ml}$ or $0.14 \mathrm{mM}$ at $\mathrm{pH} 6.5$ ), especially in relation to the high required dose (1200 mg). To enable absorption, the original amprenavir dosage 
form (Agenerase ${ }^{\circledR}$ ) consists of soft gelatin capsules containing amprenavir $(1200 \mathrm{mg})$ predissolved in a mixture of cosolvents and the surfactant $\mathrm{d}$ - $\alpha$-tocopheryl polyethyleneglycol 1000 succinate (TPGS, $2240 \mathrm{mg}$ ). Following oral administration of this formulation, Brouwers et al. (2006) collected duodenal and jejunal fluids from healthy volunteers. Mean amprenavir concentrations in the duodenum exceeded the solubilizing capacity of reference intestinal fluid up to 30-fold (Fig. 4A), demonstrating significant intraluminal solubilization. The main solubilizing excipient TPGS was present at levels well above its critical micellar concentration $(0.13 \mathrm{mM})$ (Fig. 4B). In the jejunum (ca. $90 \mathrm{~cm}$ more distally), maximum amprenavir concentrations were about 70\% lower than those in the duodenum. In a similar study with an immediate release formulation of theophylline, the concentration drop from duodenum to jejunum was even larger (ca. 90\%), suggesting a higher mucosal permeability for theophylline compared to amprenavir. For the largely hydrophilic and less permeable excipient TPGS, on the other hand, the concentration gradient between duodenum and jejunum was clearly lower (Fig. 4B).

While intraluminal concentrations are the driving force for drug permeation across the intestinal mucosa, the absorptive flux will further be affected by the permeability of the mucosa for the drug. In traditional formulation evaluation, in vitro-in vivo extrapolations therefore combine in vitro dissolution data with the estimated permeability for the active ingredient. This approach largely ignores the influence of the highly complex composition of intraluminal fluids on the drug's permeability (Kostewicz et al., in press). Obviously, intestinal concentration profiling upon drug intake enables the assessment of biorelevant drug concentrations for permeability estimation, which may be essential to elucidate the impact of transporters and enzymes on the absorption process. In addition, the potential of the drug to permeate the intestinal monolayer in relevant conditions may be evaluated starting from the intestinal fluid aspirates (Fig. 2). In this respect, intestinal fluid samples collected upon administration of amprenavir (diluted 1:1 with aqueous transport medium) were used as donor in Caco-2 transport experiments (Brouwers et al., 2006). In comparison with a plain aqueous solution of amprenavir, the intestinal fluid samples affected the amprenavir flux in multiple ways. Not only did the driving force for permeation increase as a result of high amprenavir concentrations (Fig. 4), but also the permeability was affected. Although bile salts and the excipient TPGS inhibited P-glycoprotein-based efflux of amprenavir, the overall permeability was decreased due to entrapment of amprenavir in TPGS-based micelles (Brouwers et al., 2006). The latter effect illustrates the so-called solubility-permeability interplay: food- or excipient-based strategies that enhance the solubility of lipophilic drugs may decrease their permeability by reducing the drug's partition coefficient between the membrane and the intraluminal medium. To account for these types of effects, there is a growing interest to implement permeation assessment from relevant matrices into the in vitro evaluation of absorption-enabling strategies (Kostewicz et al., in press). Intraluminal fluid sampling upon drug intake may guide the development of such tools by providing reference media.

In this framework, Vertzoni et al. (2012) collected intestinal fluids following the administration of the highly lipophilic drug danazol predissolved in the oil portion of a heterogeneous liquid meal. By ultracentrifugation of the luminal aspirates, the aqueous micellar phase was separated from a lipid phase and an interphase. Subsequently, permeation of danazol from diluted full aspirates and diluted micellar phases was studied across Caco-2 monolayers. Overall, danazol permeability decreased as a function of lipid concentration in the diluted samples (Fig. 5). In comparison with a simple aqueous medium, danazol permeability was significantly reduced when starting from diluted aspirates but not from diluted micellar phases. It should be noted that effects on permeability may be somewhat obscured due to the strong sample dilution (1/ 16) needed to ensure monolayer integrity. In the aspirates, danazol was mainly present in the lipid phase: on average, danazol concentrations were about 10 -fold higher in the aspirates than in the corresponding micellar phases. Still, the high total concentrations in the aspirates resulted in an enhanced danazol flux (thereby compensating for the reduced permeability). This suggests that, at least for danazol, the total concentration rather than the concentration in the micellar phase, should be considered the main driving force for permeation.

As illustrated above, the use of intestinal fluid samples aspirated after drug administration provides a highly relevant means of understanding the influence of intraluminal factors on drug permeation. However, the application of this approach is severely limited due to compatibility issues between the intestinal fluid and cellular-based absorption models, including Caco-2 monolayers. Usually, significant dilution is required to ensure monolayer integrity (Vertzoni et al., 2012), which obviously compromises the relevance of the obtained results. Approaches to increase the robustness of cell-based permeation models are currently under investigation (e.g. inclusion of mucus). Alternatively, tissue-based ex vivo or in situ models may be used. In this respect, Holmstock et al. (2013) successfully applied fasted and fed state human intestinal fluid pools as donor medium during the in situ perfusion of mice intestine to demonstrate reduced permeation of indinavir in postprandial conditions.

\subsection{Postprandial tablet disintegration}

Fosamprenavir is a phosphate ester prodrug of amprenavir with improved aqueous solubility. As compared to the original, solubilizing formulation of amprenavir, immediate release tablets of fosamprenavir $\left(\right.$ Telzir $^{\circledR}$ ) significantly reduce the pill burden for pa-
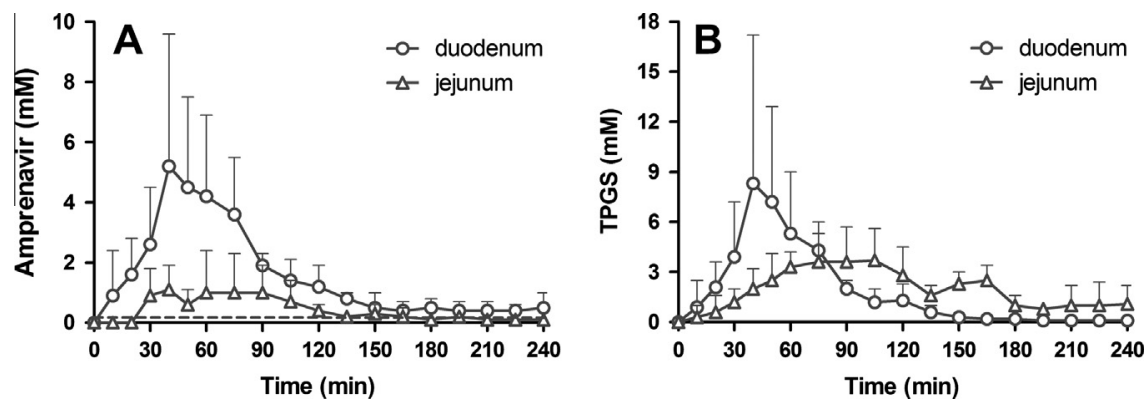

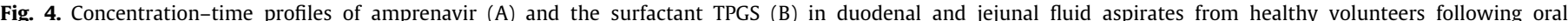

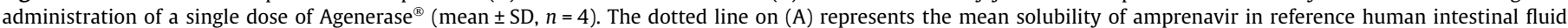
(0.18 mM). Data from Brouwers et al. (2006) and Wuyts et al. (2013). 


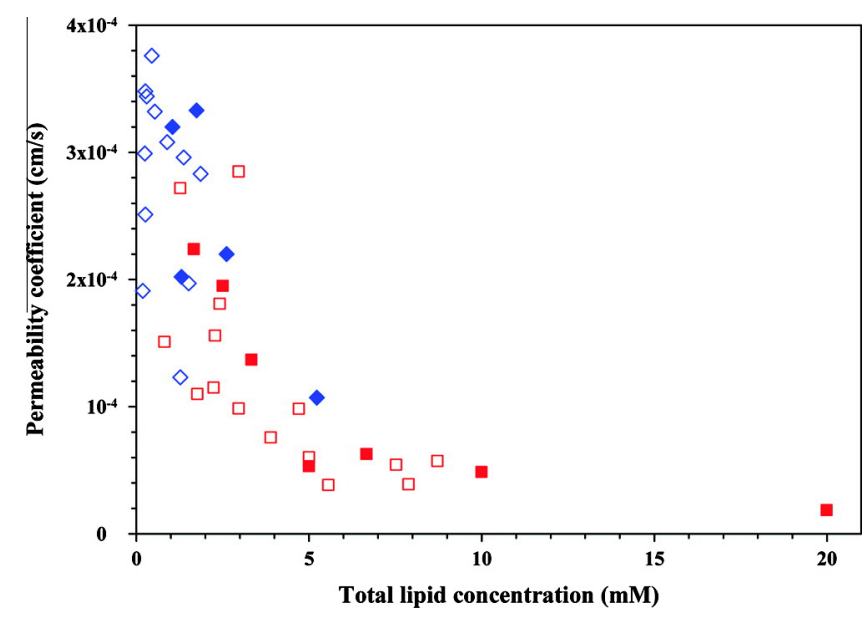

Fig. 5. Permeability of Caco-2 monolayers for the lipophilic drug danazol from intestinal fluid aspirates (squares) and their micellar phases (diamonds) as a function of the corresponding total lipid concentration. Aspirates were collected after administering a tryglyceride solution of danazol to healthy volunteers. Open symbols refer to 1:16 diluted samples; closed symbols refer to other dilutions. The total lipid concentration refers to the sum of tri-, di- and monoglycerides, fatty acids, phosphatidylcholine, lysophosphatidylcholine and cholesterol. Reprinted with permission from Vertzoni et al. (2012). Copyright 2012 American Chemical Society.

tients. Pharmacokinetic studies revealed a significant food effect on the uptake of amprenavir from fosamprenavir tablets: postprandial intake of fosamprenavir strongly delayed the onset of amprenavir absorption. For instance, upon intake of fosamprenavir with a nutritional drink, mean $t_{\max }$ increased $2.5 \mathrm{~h}$ as compared to the fasted state, without further affecting the rate or extent of absorption. Based on in vitro studies (Brouwers et al., 2007b), we hypothesized that increased phosphate concentrations in fed state conditions would inhibit the intestinal dephosphorylation of fosamprenavir, required for transepithelial permeation. To test this hypothesis in vivo, gastrointestinal fluids and plasma samples were simultaneously collected from healthy volunteers upon administration of a fosamprenavir tablet in fasted and fed state (Brouwers et al., 2007a). However, the clinical study revealed that, instead of dephosphorylation, the preceding duodenal appearance of fosamprenavir determined the onset of absorption and plasma $t_{\max }$. The strong correlation between individual plasma $t_{\mathrm{max}}$-values of amprenavir and duodenal $t_{\mathrm{max}}$-values of fosamprenavir is depicted in Fig. 6. In fed state conditions, not only duodenal appearance but also gastric dissolution of fosamprenavir appeared to be postponed as a result of an unexpected delay in gastric

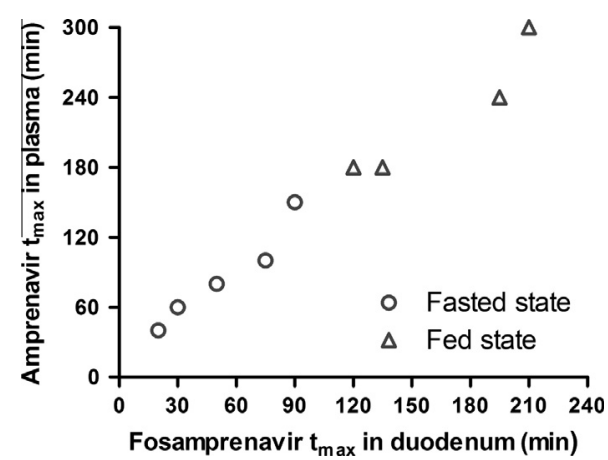

Fig. 6. Correlation between individual plasma $t_{\max }$-values of amprenavir and corresponding duodenal $t_{\mathrm{max}}$-values of fosamprenavir following oral administration of a single Telzir ${ }^{\circledR}$ tablet to healthy volunteers in fasted and fed state. Data from Brouwers et al. (2007a). disintegration of the fosamprenavir tablet. In vitro MRI studies confirmed impaired water ingress into the HPMC-coated fosamprenavir tablets in the presence of a nutritional drink (Brouwers et al., 2011), causing the immediate release tablet to behave as an enteric-coated dosage form in the postprandial stomach. From Fig. 6, it is interesting to note that not only the food-induced delay in amprenavir absorption but also the relatively high interindividual variations can be explained by differences in duodenal appearance of fosamprenavir. Overall, this study nicely demonstrates how the combination of gastrointestinal and systemic concentration profiling may assist in identifying intraluminal processes that determine absorption. In addition, the clinical data were used as reference to validate the TNO Intestinal Model (TIM-1) for evaluation of food-dependent disintegration of immediate release tablets (Brouwers et al., 2011). It appeared that TIM-1 could not only predict the impaired disintegration as such but also the impact on absorption (affecting the onset but not the extent of absorption).

\subsection{The role of acidic beverages in the gastric dissolution of weakly basic drugs}

A significant number of drugs and drug candidates are poorly water soluble weak bases. As a result of their ionization behavior, intestinal absorption often relies on dissolution in the acidic environment of the fasted state stomach. Elevated gastric $\mathrm{pH}$ due to, for instance, hypochloridia or the use of proton pump inhibitors (PPIs), may therefore compromise absorption of this type of drugs. This behavior has been confirmed by gastrointestinal concentration profiling for the antifungal drug posaconazole (Walravens et al., 2011). Upon co-administration of the oral suspension of posaconazole (Noxafil ${ }^{\circledR}$ ) with the PPI esomeprazole, the elevated gastric pH resulted in significantly lower gastric posaconazole concentrations and reduced absorption. Simultaneous intake of an acidic beverage (Coca-Cola ${ }^{\circledR}$ ) was not sufficient to completely restore the reduced posaconazole absorption, since the beverage could only partly compensate for the PPI-induced increase in gastric $\mathrm{pH}$ and decrease in posaconazole dissolution. Remarkably, posaconazole absorption was also significantly enhanced by intake with an acidic beverage in normal conditions (i.e. without PPI administration). The gastric concentration profiles revealed enhancement of posaconazole dissolution following intake with the acidic beverage instead of water; in contrast to the usually accepted hypothesis, however, this could not be attributed to an effect on the gastric $\mathrm{pH}$, in contrast to the usually accepted. From Fig. 7, it is obvious that the beverage prolonged the dissolution time window for posa-

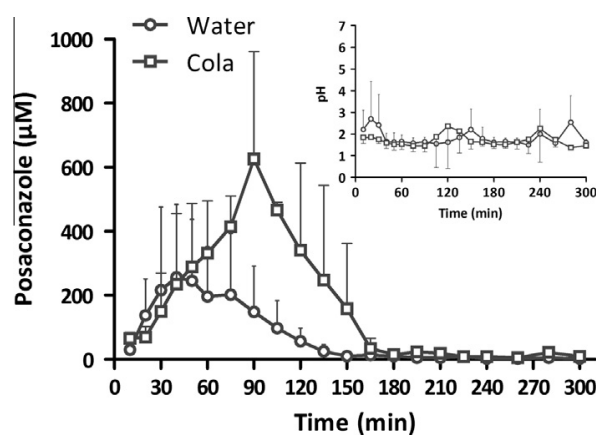

Fig. 7. Gastric concentration-time profiles of the lipophilic and weakly basic drug posaconazole following administration of the oral suspension Noxafil ${ }^{\circledR}$ to healthy volunteers. Posaconazole was administered in the fasted state with either water (circles) or the acidic beverage Coca-Cola ${ }^{\circledR}$ (squares). The inset figure displays the corresponding gastric $\mathrm{pH}$ as a function of time. Data (mean $\pm \mathrm{SD}, n=5$ ) from Walravens et al. (2011). 
conazole in the stomach, presumably by extending the gastric residence time.

\subsection{Intestinal supersaturation and precipitation of weakly basic drugs}

As already mentioned in the introduction, predicting intraluminal precipitation of drugs is currently one of the main hurdles in the design of so-called supersaturating drug delivery systems to overcome solubility-limited absorption (Bevernage et al., 2013). A few recent papers describe the use of intraluminal concentration profiling to shed light on the influence of gastrointestinal physiology on drug precipitation. Poorly soluble weak bases are the preferred model drugs, since intestinal supersaturation may simply occur upon transfer from the stomach (low pH and high solubility) to the intestine (higher $\mathrm{pH}$ and lower solubility).

A first attempt to investigate intestinal precipitation in humans involved the administration of a dispersion of the weak base gefinitib in the stomach followed by sampling of jejunal fluid using the Loc-I-Gut catheter (Bergman et al., 2007). The study could not relate differences in pharmacokinetics among the tested volunteers with the observed intraluminal behavior. In addition, the study was not able to distinguish between undissolved and precipitated drug. Although jejunal samples were analyzed with Raman spectroscopy, the solid state properties could not be determined due to interference with the complex nature of the fluids.

To avoid issues with incomplete gastric dissolution, Psachoulias et al. (2011) administered solutions of the weakly basic and lipophilic drugs ketoconazole and dipyridamole to investigate intestinal precipitation. By determining not only the drug concentration but also the drug's equilibrium solubility in the aspirated intestinal fluids, the authors were able to demonstrate supersaturation of the weak bases for up to $30 \mathrm{~min}$ (dipyridamole) and $50 \mathrm{~min}$ (ketoconazole) post-dosing. Only limited luminal precipitation was observed: compared to the total drug amount in the intestinal samples, the fraction of precipitated drug amounted to maximum $7 \%$ for dipyridamole and maximum $16 \%$ for ketoconazole. Using XRPD analysis of the aspirated ketoconazole precipitates, 3 different solid states were identified: 2 crystalline forms and 1 amorphous form as the most abundant one. Interestingly, a classic two-compartmental in vitro transfer model (Kostewicz et al., 2004) that has been designed by only considering systemic concentration data as reference, significantly overestimated the observed intestinal precipitation of dipyridamole and ketoconazole in humans. Therefore, Psachoulias et al. (2012) developed an optimized in vitro model based on the observed in vivo intraluminal data. To predict the intraluminal concentrations and to reduce in vitro precipitation, it appeared crucial to simulate drug disappearance from the duodenal compartment (due to intestinal transit and absorption of the highly permeable drugs) by implementing an outgoing flow and a reservoir compartment with concentrated biorelevant intestinal medium. Additional variables that were optimized for improved predictive power included the flow rates between the compartments, the applied hydrodynamics and the composition of simulating intestinal fluid. This setup for simulating the in vitro behavior of poorly soluble and highly permeable weak bases is one of the first in vitro models for which the development was guided by intraluminal rather than systemic in vivo data.

Similar to ketoconazole and dipyridamole, posaconazole is a weakly basic drug with poor solubility and high permeability. In the above-mentioned study on the intraluminal behavior of the posaconazole suspension Noxafil ${ }^{\circledR}$ (Section 4.2) not only gastric but also intestinal fluids were aspirated (Walravens et al., 2011). In the duodenal samples, most posaconazole was present in solid

\section{A - Intraluminal posaconazole concentrations in humans}

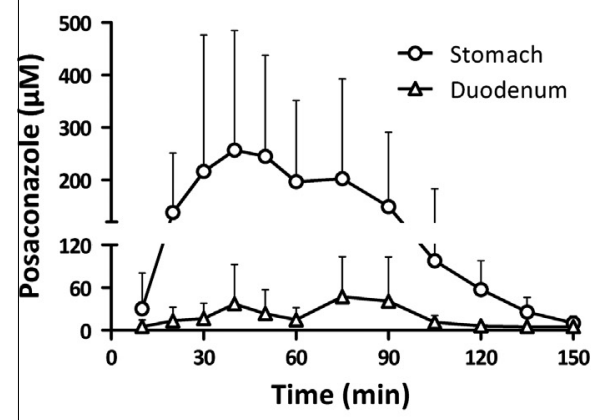

\section{B - Total versus dissolved intestinal posaconazole}

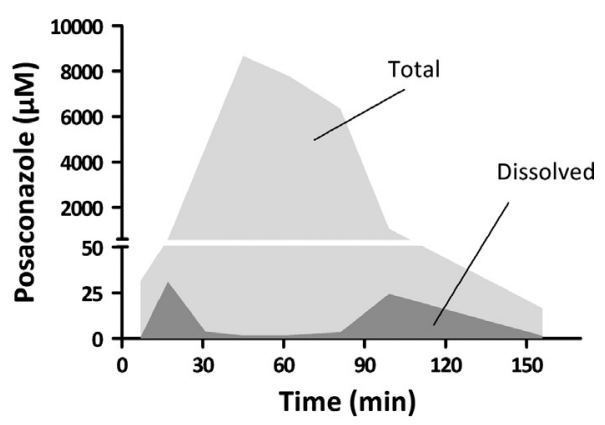

\section{C-Predicted posaconazole} concentrations in duodenum

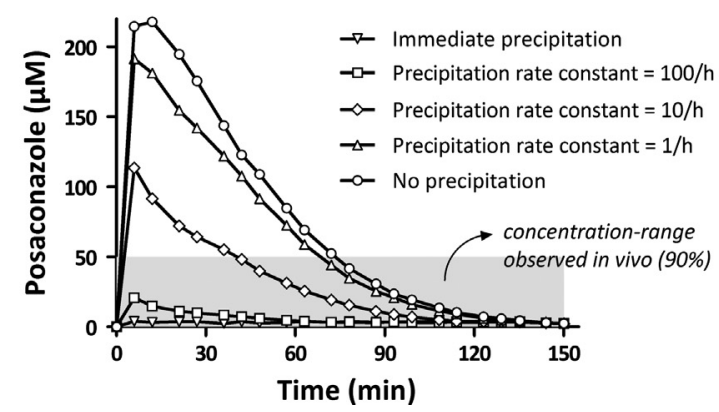

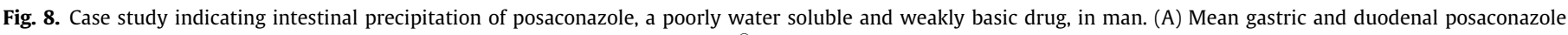

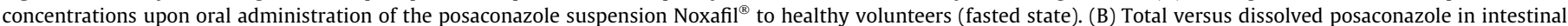

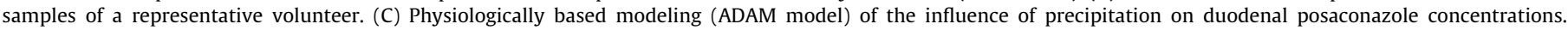
Reprinted with permission from Brouwers and Augustijns (2011). Data (A and B) from Walravens et al. (2011). 
form (Fig. 8B), but no distinction could be made between undissolved or precipitated particles. In addition, intestinal posaconazole concentrations were extremely low (at least 5- to 10 -fold lower than gastric concentrations, Fig. 8A). In an attempt to elucidate the potential role of precipitation, PBPK sensitivity analysis was performed (ADAM model), using relevant posaconazole parameters (gastric concentrations and high permeability) and varying precipitation rates as input, and duodenal concentrations as output (Brouwers and Augustijns, 2011). The modeling revealed that only the assumption of extensive posaconazole precipitation was in agreement with the low concentrations measured in humans (Fig. 8C). Most likely, the difference in precipitation between posaconazole (extensive) and dipyridamole/ketoconazole (limited) can be attributed to the difference in dosing (suspension versus solution). In case of administering a suspension, incomplete gastric dissolution may cause undissolved particles to be transferred to the intestine where they can act as nuclei for precipitation.

\section{Concluding remarks}

In contrast to systemic concentrations, gastrointestinal drug concentrations following oral administration directly reflect the intraluminal events that dictate absorption. The multitude of processes involved, combined with the highly fluctuating intraluminal environment, makes it challenging to interpret gastrointestinal concentration-time profiles. Nevertheless, the case studies discussed in this review demonstrate the added value of gastrointestinal concentration profiling in resolving poorly understood intraluminal processes, including precipitation and permeation from complex matrices. The combination with simultaneous systemic concentration assessment further helps to identify those processes determining the rate and/or extent of absorption, as well as inter-individual variability. Now that gastrointestinal concentration profiling has been established as a novel tool to evaluate intraluminal drug and formulation behavior in humans, intensified use can be expected to guide the optimization of simulation models for drug absorption. This is, for instance, manifested within the new European OrBiTo initiative that pursues the development of physiologically relevant models, supported by a solid understanding of the underlying processes. Especially for challenging drug candidates (low solubility and/or permeability) that rely on complex enabling strategies (e.g. solubilization, supersaturation), such models will allow for improved prediction of in vivo performance.

\section{Acknowledgements}

This work has received support from the Innovative Medicines Initiative Joint Undertaking (http://www.imi.europa.eu) under Grant Agreement No. 115369, resources of which are composed of financial contribution from the European Union's Seventh Framework Programme (FP7/2007-2013) and EFPIA companies' in kind contribution.

\section{References}

Bergman, E., Forsell, P., Persson, E.M., Knutson, L., Dickinson, P., Smith, R., Swaisland, H., Farmer, M.R., Cantarini, M.V., Lennernäs, H., 2007. Pharmacokinetics of gefitinib in humans: the influence of gastrointestinal factors. Int. J. Pharm. 341, 134-142.

Bevernage, J., Brouwers, J., Brewster, M.E., Augustijns, P., 2013. Evaluation of gastrointestinal drug supersaturation and precipitation: strategies and issues. Int. J. Pharm. 453, 25-35.

Bønløkke, L., Christensen, F.N., Knutson, L., Kristensen, H.G., Lennernäs, H., 1997. A new approach for direct in vivo dissolution studies of poorly soluble drugs. Pharm. Res. 14, 1490-1492.

Bønløkke, L., Hovgaard, L., Kristensen, H.G., Knutson, L., Lindahl, A., Lennernäs, H., 1999. A comparison between direct determination of in vivo dissolution and the deconvolution technique in humans. Eur. J. Pharm. Sci. 8, 19-27.
Bønløkke, L., Hovgaard, L., Kristensen, H.G., Knutson, L., Lennernäs, H., 2001. Direct estimation of the in vivo dissolution of spironolactone, in two particle size ranges, using the single-pass perfusion technique (Loc-I-Gut) in humans. Eur. J. Pharm. Sci. 12, 239-250.

Brouwers, J., Augustijns, P., 2011. Biorelevant evaluation of supersaturation and precipitation inhibition: do we understand the gastrointestinal environment? In: Bulletin Technique Gattefossé. Presented at the Journées Galéniques, SaintRémy de Provence, France, pp. 71-82.

Brouwers, J., Ingels, F., Tack, J., Augustijns, P., 2005. Determination of intraluminal theophylline concentrations after oral intake of an immediate- and a slowrelease dosage form. J. Pharm. Pharmacol. 57, 987-996.

Brouwers, J., Tack, J., Lammert, F., Augustijns, P., 2006. Intraluminal drug and formulation behavior and integration in in vitro permeability estimation: a case study with amprenavir. J. Pharm. Sci. 95, 372-383.

Brouwers, J., Tack, J., Augustijns, P., 2007a. Parallel monitoring of plasma and intraluminal drug concentrations in man after oral administration of fosamprenavir in the fasted and fed state. Pharm. Res. 24, 1862-1869.

Brouwers, J., Tack, J., Augustijns, P., 2007b. In vitro behavior of a phosphate ester prodrug of amprenavir in human intestinal fluids and in the Caco-2 system: illustration of intraluminal supersaturation. Int. J. Pharm. 336, 302-309.

Brouwers, J., Anneveld, B., Goudappel, G.-J., Duchateau, G., Annaert, P., Augustijns P., Zeijdner, E., 2011. Food-dependent disintegration of immediate release fosamprenavir tablets: in vitro evaluation using magnetic resonance imaging and a dynamic gastrointestinal system. Eur. J. Pharm. Biopharm. 77, 313-319.

Casey, D.L., Beihn, R.M., Digenis, G.A., Shambhu, M.B., 1976. Method for monitoring hard gelatin capsule disintegration times in humans using external scintigraphy. J. Pharm. Sci. 65, 1412-1413.

Corá, L.A., Américo, M.F., Oliveira, R.B., Serra, C.H.R., Baffa, O., Evangelista, R.C. Oliveira, G.F., Miranda, J.R.A., 2011. Biomagnetic methods: technologies applied to pharmaceutical research. Pharm. Res. 28, 438-455.

Perez de la Cruz, M., Moreno, M., Oth, M., Deferme, S., Lammert, F., Tack, J., Dressman, J., Augustijns, P., 2006. Characterization of fasted-state human intestinal fluids collected from duodenum and jejunum. J. Pharm. Pharmacol. $58,1079-1089$

Go, V.L., Hofmann, A.F., Summerskill, W.H., 1970. Simultaneous measurements of total pancreatic, biliary, and gastric outputs in man using a perfusion technique. Gastroenterology 58, 321-328.

Holmstock, N., Bruyn, T.D., Bevernage, J., Annaert, P., Mols, R., Tack, J., Augustijns, P. 2013. Exploring food effects on indinavir absorption with human intestinal fluids in the mouse intestine. Eur. J. Pharm. Sci. 49, 27-32.

Jobin, G., Cortot, A., Godbillon, J., Duval, M., Schoeller, J.P., Hirtz, J., Bernier, J.J., 1985 Investigation of drug absorption from the gastrointestinal tract of man. I Metoprolol in the stomach, duodenum and jejunum. Br. J. Clin. Pharmacol. 19 (Suppl. 2), 97S-105S.

Kalantzi, L., Goumas, K., Kalioras, V., Abrahamsson, B., Dressman, J.B., Reppas, C. 2006. Characterization of the human upper gastrointestinal contents under conditions simulating bioavailability/bioequivalence studies. Pharm. Res. 23, $165-176$.

Knutson, L., Odlind, B., Hällgren, R., 1989. A new technique for segmental jejuna perfusion in man. Am. J. Gastroenterol. 84, 1278-1284.

Knutson, T., Fridblom, P., Ahlström, H., Magnusson, A., Tannergren, C., Lennernäs, H., 2009. Increased understanding of intestinal drug permeability determined by the LOC-I-GUT approach using multislice computed tomography. Mol. Pharm. 6 $2-10$.

Kostewicz, E.S., Wunderlich, M., Brauns, U., Becker, R., Bock, T., Dressman, J.B., 2004 Predicting the precipitation of poorly soluble weak bases upon entry in the small intestine. J Pharm Pharmacol 56, 43-51.

Kostewicz, E.S., Aarons, L., Bergstrand, M., Bolger, M.B., Galetin, A., Hatley, O., Jamei M., Lloyd, R., Pepin, X., Rostami, A., Sjögren, E., Tannergren, C., Turner, D.B., Wagner, C., Weitschies, W., Dressman, J., 2013a. PBPK models for the prediction of in vivo performance of oral dosage forms. Eur J Pharm Sci, in press doi:10.1016/j.ejps.2013.09.008.

Kostewicz, E.S., Abrahamsson, B., Brewster, M., Brouwers, J., Butler, J., Carlert, S., Dickinson, P.A., Dressman, J., Holm, R., Klein, S., Mann, J., McAllister, M., Minekus, M., Muenster, U., Müllertz, A., Verwei, M., Vertzoni, M., Weitschies, W. Augustijns, P., 2013b. In vitro models for the prediction of in vivo performance of oral dosage forms. Eur. J. Pharm. Sci., in press doi:10.1016/j.ejps.2013.08.024.

Koziolek, M., Garbacz, G., Neumann, M., Weitschies, W., 2013. Simulating the postprandial stomach: physiological considerations for dissolution and release testing. Mol. Pharm. 10, 1610-1622.

Lennernäs, H., 1998. Human intestinal permeability. J. Pharm. Sci. 87, 403-410.

Lennernäs, H., Aarons, L., Augustijns, P., Beato, S., Bolger, M., Box, K., Brewster, M. Butler, J., Dressman, J., Holm, R., Julia Frank, K., Kendall, R., Langguth, P., Sydor, J., Lindahl, A., McAllister, M., Muenster, U., Müllertz, A., Ojala, K., Pepin, X., Reppas, C., Rostami-Hodjegan, A., Verwei, M., Weitschies, W., Wilson, C., Karlsson, C., Abrahamsson, B., 2013. Oral biopharmaceutics tools - time for a new initiative - an introduction to the IMI project OrBiTo. Eur. J. Pharm. Sci., in press doi:10.1016/j.ejps.2013.10.012.

Longstreth, G.F., Malagelada, J.R., Go, V.L., 1975. The gastric response to a transpyloric duodenal tube. Gut 16, 777-780.

McAllister, M., 2010. Dynamic dissolution: a step closer to predictive dissolution testing? Mol. Pharm. 7, 1374-1387.

Näslund, E., Bogefors, J., Grybäck, P., Jacobsson, H., Hellström, P.M., 2000. Gastric emptying: comparison of scintigraphic, polyethylene glycol dilution, and paracetamol tracer assessment techniques. Scand. J. Gastroenterol. 35, 375379. 
Petri, N., Tannergren, C., Holst, B., Mellon, F.A., Bao, Y., Plumb, G.W., Bacon, J. O'Leary, K.A., Kroon, P.A., Knutson, L., Forsell, P., Eriksson, T., Lennernas, H., Williamson, G., 2003. Absorption/metabolism of sulforaphane and quercetin, and regulation of phase II enzymes, in human jejunum in vivo. Drug Metab. Dispos. 31, 805-813.

Psachoulias, D., Vertzoni, M., Goumas, K., Kalioras, V., Beato, S., Butler, J., Reppas, C., 2011. Precipitation in and supersaturation of contents of the upper small intestine after administration of two weak bases to fasted adults. Pharm. Res. 28, 3145-3158.

Psachoulias, D., Vertzoni, M., Butler, J., Busby, D., Symillides, M., Dressman, J. Reppas, C., 2012. An in vitro methodology for forecasting luminal concentrations and precipitation of highly permeable lipophilic weak bases in the fasted upper small intestine. Pharm. Res. 29, 3486-3498.

Read, N.W., Al Janabi, M.N., Bates, T.E., Barber, D.C., 1983. Effect of gastrointestinal intubation on the passage of a solid meal through the stomach and small intestine in humans. Gastroenterology 84, 1568-1572.

Rees, W.D., Go, V.L., Malagelada, J.R., 1979. Simultaneous measurement of antroduodenal motility, gastric emptying, and duodenogastric reflux in man. Gut 20, 963-970.

Schiller, C., Fröhlich, C.-P., Giessmann, T., Siegmund, W., Mönnikes, H., Hosten, N. Weitschies, W., 2005. Intestinal fluid volumes and transit of dosage forms as assessed by magnetic resonance imaging. Aliment Pharmacol. Ther. 22, 971979.
Tannergren, C., Knutson, T., Knutson, L., Lennernäs, H., 2003. The effect of ketoconazole on the in vivo intestinal permeability of fexofenadine using a regional perfusion technique. Br. J. Clin. Pharmacol. 55, 182-190.

Vertzoni, M., Markopoulos, C., Symillides, M., Goumas, C., Imanidis, G., Reppas, C., 2012. Luminal lipid phases after administration of a triglyceride solution of danazol in the fed state and their contribution to the flux of danazol across caco-2 cell monolayers. Mol. Pharm. 9, 1189-1198.

Walravens, J., Brouwers, J., Spriet, I., Tack, J., Annaert, P., Augustijns, P., 2011. Effect of $\mathrm{pH}$ and comedication on gastrointestinal absorption of posaconazole: monitoring of intraluminal and plasma drug concentrations. Clin. Pharmacokinet. 50, 725-734.

Weitschies, W., Wilson, C.G., 2011. In vivo imaging of drug delivery systems in the gastrointestinal tract. Int. J. Pharm. 417, 216-226.

Weitschies, W., Blume, H., Mönnikes, H., 2010. Magnetic marker monitoring: high resolution real-time tracking of oral solid dosage forms in the gastrointestinal tract. Eur. J. Pharm. Biopharm. 74, 93-101.

Wilding, I.R., Coupe, A.J., Davis, S.S., 2001. The role of gamma-scintigraphy in oral drug delivery. Adv. Drug Deliv. Rev. 46, 103-124.

Wuyts, B., Brouwers, J., Mols, R. Tack, J., Annaert, P., Augustijns, P. 2013, Solubility profiling of HIV protease inhibitors in human intestinal fluids. J. Pharm. Sci. 102, 3800-3807. 